\section{One year of EPO markedly increases HDL cholesterol levels in predialysis CKD patients}

Data indicate that abnormal serum lipid parameters-for example, decreased levels of HDL cholesterol—might contribute to the high incidence of cardiovascular complications in patients with chronic kidney disease (CKD). A previously published randomized controlled trial showed that administration of recombinant human erythropoietin (EPO) during the early stages of CKD slows disease progression. Now, in a nested sub-study of the original trial, the authors have examined how EPO influences serum lipid profiles in the predialysis CKD population.

Forty-five of 88 patients with stage 3 or 4 CKD were randomly assigned to receive subcutaneous epoetin alfa $(50 \mathrm{U} / \mathrm{kg} /$ week, with a hemoglobin target of at least $130 \mathrm{~g} / \mathrm{l})$. The remaining 43 patients received epoetin alfa only if their hemoglobin level dropped below $90 \mathrm{~g} / \mathrm{l}$.

At the end of the 12-month study period, serum levels of total cholesterol, LDL cholesterol and triglycerides had declined markedly in both groups. Only in the group treated with EPO weekly were concentrations of serum $\mathrm{HDL}$ cholesterol significantly increased $(P<0.001)$. This improvement was positively correlated with hemoglobin concentration, which increased to target levels $(P<0.001$ compared with baseline). A marked decrease in atherogenic LDL:HDL ratio was detected only in the cohort of patients who received EPO each week. Because renal function (measured as serum creatinine level and creatinine clearance) was more effectively preserved in this group, the authors suggest that the beneficial impact of early EPO treatment on HDL cholesterol level might contribute to amelioration of CKD progression.

Original article Siamopoulos KC et al. (2006) Long-term treatment with EPO increases serum levels of high-density lipoprotein in patients with CKD. Am J Kidney Dis 48: 242-249

\section{Extracorporeal blood purification does not prevent contrast nephropathy}

Radiocontrast-induced nephropathy (RCIN) is a primary cause of hospital-acquired acute renal injury. Excretion of radiocontrast agent from the kidneys is delayed in patients with underlying renal disease. It has been proposed that improving clearance through treatment with extracorporeal blood purification (EBP) could prevent RCIN, but trial data are conflicting.

Cruz et al. systematically reviewed available literature through a search of PubMed, the Cochrane Collaboration Database, EMBASE and CINAHL, as well as reviewing retrieved bibliographies and consulting experts. They identified six randomized controlled trials and two nonrandomized trials comparing EBP with standard therapy ( $n=412)$ - six used hemodialysis, one continuous venovenous hemofiltration, and one continuous venovenous hemodiafiltration, immediately before, during, or within $2 \mathrm{~h}$ of administration of contrast agents. RCIN incidence, defined as an increase in serum creatinine concentration of at least $0.5 \mathrm{mg} / \mathrm{dl}$ $(44 \mu \mathrm{mol} / \mathrm{l})$, was $35.2 \%$ with standard medical therapy (e.g. saline, calcium channel blockers, aminophylline), and $27.8 \%$ with EBP. RCIN incidence was not significantly reduced with EBP compared with standard therapy, but the results of the trials were heterogeneous. When only trials studying hemodialysis were included, there was a nonsignificant trend favoring standard therapies over periprocedural EBP. Form of periprocedural treatment did not markedly affect the need for acute temporary renal replacement therapy, or the combined incidence of death and/or the need for permanent renal replacement therapy.

Additional studies with alternative endpoints and investigating newer techniques are warranted, but these results indicate that periprocedural EBP does not decrease RCIN incidence compared with standard medical therapy.

Original article Cruz DN et al. (2006) Extracorporeal blood purification therapies for prevention of radiocontrastinduced nephropathy: a systematic review. Am J Kidney Dis 48: $361-371$

\section{Cardiovascular risk factors under-managed in advanced type 2 diabetic nephropathy}

A recent study indicates that although patients with advanced diabetic nephropathy are at high risk of cardiovascular disease and its associated morbidity and mortality, traditional cardiovascular risk factors are being under-treated in these 